\title{
Significance Analysis of Vehicle Operation Modes for Pollutant Emission at a Signalized Intersection
}

\author{
Yuanyuan Fan ${ }^{1,}$, Janice Daniel ${ }^{1, b}$ \\ ${ }^{1}$ New Jersey Institute of Technology \\ 323 Martin Luther King Boulevard, Newark, NJ, US \\ ayf42@njit.edu, ${ }^{b}$ daniel@njit.edu
}

\begin{abstract}
Keywords: Vehicle emission estimation, Vehicles activity, Operation modes, VISSIM, MOVES, Multiple linear regression model, Signalized intersection, Significance evaluation

Abstract. In this paper, we developed vehicle emissions models as a function of the five operation modes of vehicles at a signalized intersection. Using VISSIM simulation software, second-by-second running activities data of the vehicles were generated for a signalized intersection. The outputs from VISSIM include were used to identify the operation modes of the vehicles and used as input into the EPA emissions model MOVES. The emissions $\mathrm{CO}$ was estimated on hourly level. Finally, based on the obtained operation mode data, $\mathrm{CO}$ pollutant emission regression model was established. The model also indicate the significance of the operation modes on the $\mathrm{CO}$ emission and provides a basis for understanding the contribution of the operation mode on pollutant emissions.
\end{abstract}

\section{Introduction}

It has been shown that CO emission in urban area is as high as $90 \%$ of the total amount [1], among which vehicle emissions is a primary factor. About two-thirds of the total emissions of $\mathrm{CO}$ come from transportation sector. In order to effectively control vehicle emissions, it is essential to understand the quantitative relationship between vehicle operational modes (i.e. Braking, Idling, Cruise/Acceleration, Low Speed Coasting, and Moderate Speed Coasting) and the pollutant emissions [2].

Several vehicle emissions estimation models have been developed in recent years. The second generation EPA vehicle emissions model is called Motor Vehicle Emission Models (MOVES). It was developed by EPA's Office of Transportation and Air Quality (OTAQ) in 2002 and first released to the public in 2010. MOVES serves both the state and local agencies and it is capable of estimating 59 pollutants from 13 vehicle classes, 5 source types and 5 road types.

Research has shown that emission results are sensitive to the operation modes of the vehicles [3]. Operation mode represent the portion that vehicles taken in the overall profile. For this reason, emission estimations tools such as MOVES utilize vehicle activity data to complete the emission study. Critical to the accuracy of the emissions estimation is how the vehicle activity data is collected. The existing research on vehicle emissions models discusses three types of approaches for collecting traffic activity data for emissions study. These approaches include: using simulation software [4]; using lab experiment data [5]; and using sensors to measure field data [6]. There are distinct advantages and disadvantages for using different sources of data. On-board emission technology is capable of collecting field emission data, but the cost is relatively high and limited data is obtained at one time. The use of remote sensing technology also has difficulty to collect multiple vehicle activity data at one time. Individual vehicle activity data or limited vehicle activity data is difficult for use in describing air quality performance influenced by the vehicle operation modes. On the contrary, the cost of simulation-based approach is relatively low. In addition, the functionality of the simulation software enables generating simultaneous estimates of emissions by importing the simulated vehicle trajectory data. For this reason, vehicle activity data from simulation becomes the first choice for data collection in this study. 


\section{Literature Review}

There are numerous studies estimating and predicting vehicle emissions at intersections in recent years. The most prominent factors associated with vehicles emissions in the intersection system can be classified into four categories. These categories include: traffic related, vehicles mode and year related, traffic control strategies related, and others factors such as season and weather.

Abou-Senna and Radwan (2013) [7] studied the relationship of vehicle emission and speed. They found that with the increasing of speed from $20 \mathrm{mph}$ to $80 \mathrm{mph}, \mathrm{CO} 2$ emission first decreased and then increased, and at the speed of $60 \mathrm{mph}$, there is a minimum emission appeared. In their study, VISSIM and MOVES were utilized to generate the traffic data and the corresponding emissions, respectively. The results were then analyzed using stepwise regression.

Zhang et al. (2014) [8] established a model to evaluate emissions due to acceleration at an intersection. Field data were collected at an intersection during the peak hour. GPS data were also collected from passenger cars about $2500 \mathrm{ft}$ away from the intersection. Acceleration models were built to calculate the instantaneous speed.

Stanek and Breiland (2013) [9] estimated Green House Gas (GHG) emission at two lane roadway intersections. The concept of this research is that fuel and emissions are convertible since carbon amounts can be measured both in polluted emissions and in fuel. Thus, the research uses fuel consumption to present emission based on demand volume. The authors sought to find out how different control types would affect vehicle emission and the fuel consumption. The analysis assumes an intersection of 2500 feet with four legs. There are fifty-four groups of variables are designed as inputs for the simulation model to obtain emission outputs, of which each group of variables include intersection daily volume, volumes of major and minor streets, and turning traffic percentages. Multivariable regression method was used to analyze the relationships between fuel consumption and key variables. The study found that among these variables, the vehicle volume is the most significant variable for all the regressions models. It also shows major $\&$ minor streets splits are not prominent for all models.

\section{Data processing}

The data processing for the research is presented in the flowchart shown in Figure 1.

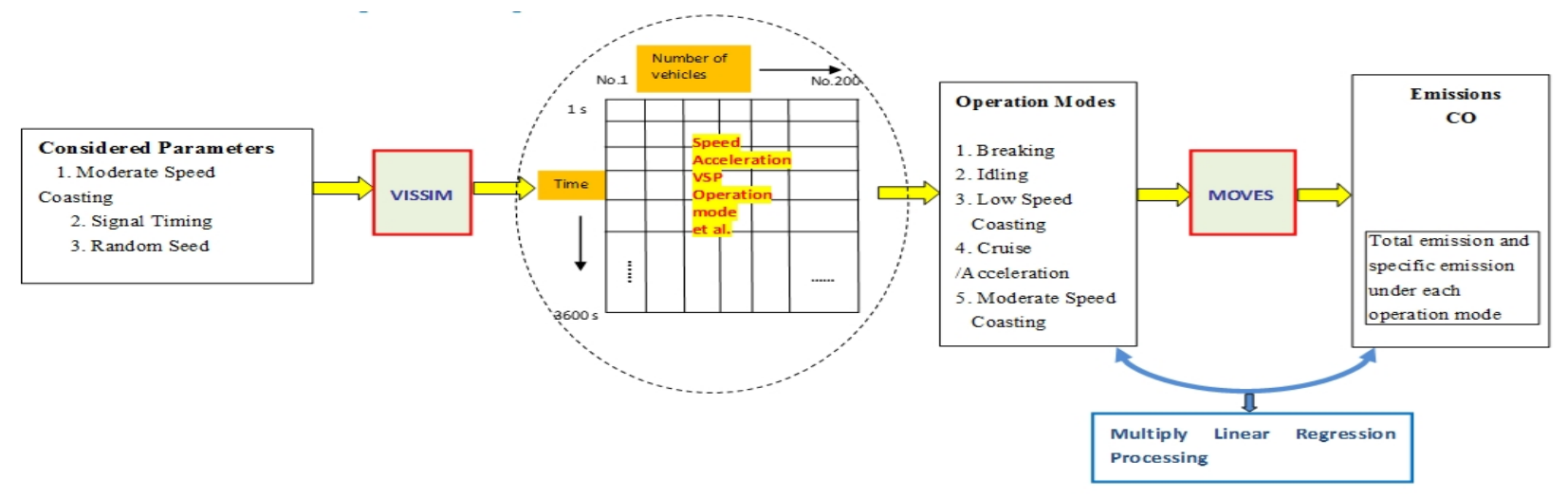

Fig. 1 Data processing flowchart

The process involves first modeling vehicle activity for an isolated intersection in VISSIM at a variable vehicular volume. Random seed numbers vary from 1 to 40 . The output of VISSIM is the speed and acceleration for each vehicle at every second during a one hour time period. Based on the definition of vehicle operation model, the percentage of time all vehicles spent in each of the five operation modes, i.e., Braking, Idling, Cruise/Acceleration, Low Speed Coasting, and Moderate Speed Coasting is determined and used as an input into MOVES. Next, using MOVES we get the emission of CO. Finally, a regression analysis was performed to develop the relationship between the operation modes and the $\mathrm{CO}$ emission. 
VISSIM is used in generating the vehicle activity data used in the development of the emissions model. The input parameters of VISSIM include vehicle volume and signal timing, turning percentage, road characteristics, vehicle characteristics, driving behavior and desired speed. Among these inputs, the first two parameters, vehicle volume and signal timing, are adjustable and the remaining variables used fixed values for each simulation run in this study. The output of VISSIM includes speed, acceleration, delay time, in-queue, dwelling time and number of stops. The advantage of using VISSIM in this research is that we can obtain speed and acceleration of every vehicle at every second within one hour. This data will directly be used in estimating vehicle emission models and in categorizing the operation mode of the vehicle. Using the data obtained from VISSIM, the percentage of time each vehicle spends in each of the five operation modes is imported into MOVES and the corresponding emissions of various pollutants is determined.

\section{Methodology}

The roadway used in this research is a four-lane urban unrestricted access road with signalized intersections, as shown in Figure 2. The length of the approaches to the intersection varies between 390 $\mathrm{ft}$ and $1460 \mathrm{ft}$. It is a 4 lane roadway with the lane width of 12 feet wide.

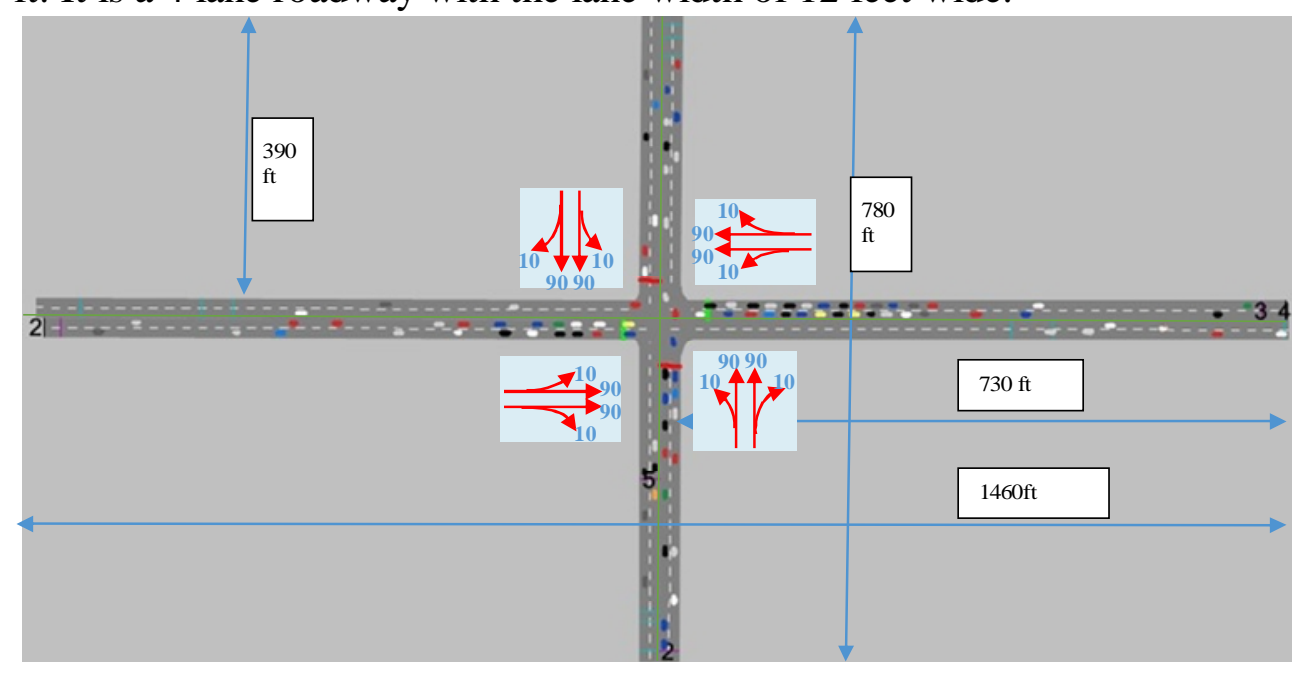

Figure 2 Roadway identification and simulation in VISSIM.

VISSIM is used to generate data of on-road vehicles at the trajectory level. The input of the VISSIM includes operation time, number of vehicles, signal timing, random seed value and geometric-related parameters for generating the vehicles' simulation runs. A simulation time of 3600 seconds is used for each simulation run; the volume of vehicles is selected to be $800 \mathrm{vph}$ for the entering volume to the intersection. The outcome data of VISSIM includes the speed and acceleration of every vehicle for every second, which is called the activity data that will be used to categorize the operation mode.

To estimate emissions quantities MOVES was used. One of the inputs to MOVES is the frequency of time vehicles spend in each operation mode. In order to find the mode frequency, vehicle specific power (VSP) was calculated. VSP is a useful parameter that can represents the amount of power that a unit weight of vehicle requires to overcome rolling resistance, engine force and air force. The EPA introduced the VSP as an important influential factor for the vehicle emissions when it became clear that speed alone could not fully reveal the relationship between vehicle emissions and the vehicle's performance [10]. Each vehicle's VSP and second-by-second speed are combined and used to calculate the respective percentages of five operation modes. These modes include Braking, Idling, Cruise/Acceleration, Low Speed Coasting, and Moderate Speed Coasting. 


\section{Model Development AND RESULTS}

Vehicle emission model was developed using multiple regression to estimate the relationship between pollutant emission and various operation modes. Five operation modes were taken as the independent variables with the operation modes denoted as vector $\mathbf{X}=\left[1, x_{1}, x_{2}, \mathrm{~L}, x_{m}\right]$, where $\mathrm{m}$ is represented as the number of the independent variables, i.e., $\mathrm{m}=1$ to 5 . The $\mathrm{CO}$ emission is the independent variable and is denoted as $\mathbf{Y}$. $X$ and $Y$ satisfy the following linear regression model [11]:

$$
\mathbf{Y}=\mathbf{X} \boldsymbol{\beta}+\boldsymbol{\varepsilon} .
$$

Where $\boldsymbol{\beta}$ is the matrix coefficient, $\boldsymbol{\varepsilon}$ refers to random noise vector. Then F-distribution test was used to test whether the obtained linear regression model is significant [12].

Unstandardized Coefficient Estimates. Based on the methodology discussed above, the vehicle emissions model was developed. In the first step of the model development, unstandardized regression coefficients were developed and shown in Table 1. Unstandardized regression coefficient reflects the impact of the independent variable (one of the five operation modes) on $\mathrm{CO}$ emission, when the variables increase by 1 unit.

Table 1 Coefficients of Regression Model

\begin{tabular}{|l|l|l|l|l|l|l|}
\hline \multirow{2}{*}{ Vehicle volume } & \multirow{3}{*}{ Unstandardized regression coefficients of five operation modes } \\
\cline { 3 - 6 } & & $\begin{array}{l}\text { Braking } \\
\left(\mathbf{X}_{\mathrm{B}}\right)\end{array}$ & $\begin{array}{l}\text { Idling } \\
\left(\mathbf{X}_{\mathrm{I}}\right)\end{array}$ & $\begin{array}{l}\text { Low Speed } \\
\text { Coasting } \\
\left(\mathbf{X}_{\mathrm{L}}\right)\end{array}$ & $\begin{array}{l}\text { Cruise } \\
\text { /Acceleration } \\
\left(\mathbf{X}_{\mathrm{C} / \mathrm{A})}\right.\end{array}$ & $\begin{array}{l}\text { Moderate } \\
\text { Speed Coasting } \\
\left(\mathbf{X}_{\mathrm{M}}\right)\end{array}$ \\
\hline
\end{tabular}

CO Emission Model. The obtained vehicle emissions model with unstandardized regression coefficients for the $\mathrm{CO}$ emission of is as following:

$$
\text { Emission }_{C O}=-23.344 X_{B}-12.564 X_{I}+6.410 X_{L}+52.575 X_{C / A}-73.424 X_{M} \text {. }
$$

From equation (2), we can find that the $\mathrm{CO}$ emission can be estimated by Braking, Idling, Low speed coasting, Cruise/acceleration, and Moderate speed coasting. Among these five variables, Low speed coasting, and Cruise/acceleration have positive coefficients for the $\mathrm{CO}$ emission, which indicates that these operation modes have positive co-relationship with the $\mathrm{CO}$ emission. On the other hand, the other modes of Braking, Idling and moderate coasting have negative coefficients for the $\mathrm{CO}$ emission, which shows that these three operation modes have negative co-relationship with the $\mathrm{CO}$ emission. It indicates that, the larger the percentages of Braking, Idling and Moderate speed coasting, the lower the $\mathrm{CO}$ emission. Furthermore, Moderate speed coasting has the biggest absolute coefficient, which indicates that Moderate speed coasting has the most significant influence on the $\mathrm{CO}$ emission. In actual application, if more vehicles operate in the Moderate speed mode, which indicates VSP $<0$ and $25 \leq$ Speed $<50$, the $\mathrm{CO}$ emission can be significantly decreased.

\section{Conclusions}

In this research, the relationship between the vehicle operation modes and the emissions of pollutants is established. First, we use VISSIM software to produce the speed and the acceleration of 800 cars (for vehicle volume of 200) at every second during one hour. Second, through calculation, the percentages of five operation modes of all vehicles are obtained, which is further used as input into MOVES software and the emissions of four pollutants are generated. Third, we establish the linear regression models on the relationship between the five operation modes and the $\mathrm{CO}$ emission. The finding and model provides directions to traffic authority on how to minimize vehicle emissions in the future's study. 


\section{References}

[1] Environmental Protection Agency- Six Principal Pollutants, http://www.epa.gov/airtrends/, Accessed in 2013.

[2] EPA, Methodology for developing model emission rates for EPA'S multy-scale motor vehicle and equipment emission system, October 2002

[3] LeBlanc, David C., et al. "Driving pattern variability and impacts on vehicle carbon monoxide emissions." Transportation Research Record 1472, 1995

[4] Abou-Senna, Hatem, and Essam Radwan. "VISSIM/MOVES integration to investigate the effect of major key parameters on $\mathrm{CO}<\mathrm{sub}>2</ \mathrm{sub}>$ emissions." Transportation Research Part D: Transport and Environment 21 (2013): 39-46.

[5] Djoric, Vladimir, Jadranka Jovic, and Smiljan Vukanovic. "Collection and assessment of instantaneous vehicle emissions on street network." Transport Research Arena (TRA) 5th Conference: Transport Solutions from Research to Deployment. 2014.

[6] Jimenez-Palacios, Jose Luis. Understanding and quantifying motor vehicle emissions with vehicle specific power and TILDAS remote sensing. Diss. Massachusetts Institute of Technology, 1998.

[7] Hatem Abou-Senna and Essam Radwan. Developing a Microscopic Transportation Emissions Model to Estimate Carbon Dioxide Emissions on Limited-Access Highways. Journal of the Transportation Research Board, No. 2428, Transportation Research Board of the National Academies, Washington,D.C., 2014, pp. 44-53. DOI: 10.3141/2428

[8] Zhang, Shaojun, et al. "Historic and future trends of vehicle emissions in Beijing, 1998-2020: A policy assessment for the most stringent vehicle emission control program in China." Atmospheric Environment 89 (2014): 216-229.

[9] Stanek, David, and Chris Breiland. "Quick Estimation Method for Greenhouse Gas Emissions at Intersections." Transportation Research Board 92nd Annual Meeting. No. 13-1428. 2013.

[10] EPA 2014 , https://www3.epa.gov/otaq/models/moves/index.htm

[11]. Kutner, Michael H., Chris Nachtsheim, and John Neter. Applied linear regression models. McGraw-Hill//rwin, 2004.

[12] Neter, John, William Wasserman, and Michael H. Kutner. "Applied regression models." Homewood, IL: Irwin, 1989. 